\section{MUC5B: a good target for future therapy in pulmonary fibrosis?}

\author{
Rahul Mahida, Alice M Turner
}

Idiopathic pulmonary fibrosis (IPF), sarcoidosis and systemic sclerosis can each cause diffuse parenchymal lung disease involving alveolar epithelial abnormalities, and manifest as interstitial lung fibrosis. Systemic sclerosis is an autoimmune connective tissue disease and sarcoidosis is a multisystem granulomatous disease; the development of lung fibrosis in both conditions generally indicates a poor prognosis. IPF is a progressive disease which is thought to occur after recurrent injury to the alveolar epithelium followed by dysregulation of cellular homoeostasis, abnormal repair and excessive deposition of extracellular matrix, ultimately leading to loss of lung function. ${ }^{1}$ Mortality $3-5$ years after diagnosis of IPF is $50 \%$, and treatment options are currently limited.

Seibold et $a l^{2}$ have previously shown that a variant single-nucleotide polymorphism (SNP) in the promoter region of an airway mucin gene (MUC5B) is associated with greatly increased production of MUC5B, and with pulmonary fibrosis. This suggested that dysregulated MUC5B expression in the lung may be key to the pathogenesis of pulmonary fibrosis. One hypothesis is that an excess of MUC5B causes bronchiolar plugging and impairs mucosal host defence, reducing clearance of inhaled substances (micro-organisms, cigarette smoke, etc), resulting in a chronic inflammatory burden on the alveolar surface, and, hence, development of IPF. ${ }^{2}$ Alternatively, MUC5B could interfere with alveolar repair, either by interfering with the interaction between the type II alveolar epithelial cells and the underlying matrix, or by interfering with the surface-tension properties of surfactant. This could enhance alveolar collapse and fibrosis of adjacent bronchoalveolar units, and eventually lead to the development of IPF. ${ }^{3}$

The genetic association study by Stock et $a l^{4}$ confirms the findings published in 2011 by Seibold et $a l^{2}$ and Zhang et $\mathrm{al}^{5}$ that a single SNP (rs35705950) in the promoter region of $M U C 5 B$ predisposes to IPF. The MUC5B finding in IPF exceeds

Centre for Translational Inflammation Research, University of Birmingham, QEHB, Birmingham, UK

Correspondence to Alice M Turner, Centre for Translational Inflammation Research, University of Birmingham, QEHB, Mindelsohn Way, Birmingham B15 2WB, UK; a.m.wood@bham.ac.uk genome-wide significance levels, and replicates prior work that was supported by an attempt at explaining the functional role of the SNP, thus, while not novel, this finding is almost incontrovertible. Following adjustment for disease severity, a trend bordering on statistical significance was observed by Stock et al between the MUC5B variant and slower decline in forced vital capacity in patients with IPF, which may be of interest clinically if it implies a better prognosis after development of disease. Similar association analyses with pulmonary fibrosis in sarcoidosis and scleroderma patients were largely negative, and confirm the findings of Peljito et al. ${ }^{6}$

There are some limitations to their work. First, variations in treatment within all groups could have impacted on the decline analyses. Second, the small cohort sizes for the studies regarding systemic sclerosis and sarcoidosis made these analyses underpowered, and no replication cohort was assessed. While the authors comment that another genetic association study ${ }^{6}$ also had similar results, their patient group was smaller and, therefore, more underpowered, making it difficult to be confident of the lack of association reported. Assuming 100\% genotyping success rate, Stock et al had over 80\% power to detect a SNP conferring an OR of 1.7 in the scleroderma analysis, and 2.5 in the sarcoidosis analysis. While it is arguable that these ORs are less than that conferred by the SNP in the original IPF work, they are significantly higher than the gold standard for genetic association work. Further studies using larger patient cohorts of non-IPF patients are, therefore, required to confirm the findings reported here.

Given that this gene has rarely been considered in non-IPF, and no functional work is included in this manuscript, it remains possible that the true functional variant lies away from rs35705950. This SNP could be acting as a marker for another polymorphism with which it is in linkage disequilibrium (LD); such unscreened genetic variants, particularly in repetitive regions which the authors were unable to assess, may be the true disease-associated variant. A broader approach to the gene rather than a single SNP analysis (eg, haplotype tagging algorithm) could be used to investigate this further. Alternatively, evaluation of MUC5B expression in lung tissue of patients with lung fibrosis in the context of systemic sclerosis and sarcoidosis could be employed. Overexpression could suggest involvement of genetic variants within, or in LD with $M U C 5 B$, while the absence of MUC5B overexpression would highlight MUC5B and related pathways as IPF-specific, rather than an end pathway of all fibrotic lung disease.

Although identification of the SNP rs35705950 could be used to target people at risk for developing IPF, and perhaps at lower risk of decline, the wider implication of this finding is that mucins may be important aetiologic factors in IPF, which could refocus future therapeutic studies in interstitial lung disease towards lung mucins, and communication between bronchiolar and alveolar surfaces. Further investigation is required into the effects of the MUC5B variant on secretion rates of MUC5B, and the expression of MUC5B in alveolar epithelia in patients with this genetic variation. Finally, agents that reduce MUC5B transcriptional activity in vitro should be tested for activity in vivo, including in subjects expressing the variant genotype. ${ }^{2}$

Contributors RM and AMT drafted the manuscript; AMT acts as guarantor.

Funding AMT has current funding from the Wellcome Trust, NIHR HTA (grant number 11/27/01), Alpha 1 Foundation, UHB charities and Linde REALfund; none of her funders had any input to this manuscript.

Competing interests None.

Provenance and peer review Commissioned; internally peer reviewed.

To cite Mahida R, Turner AM. Thorax 2013;68:401.

Published Online First 17 January 2013

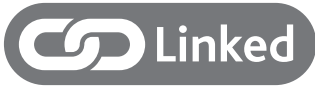

- http://dx.doi.org/10.1136/thoraxjnl-2012-201786

Thorax 2013:68:401.

doi:10.1136/thoraxinl-2012-202957

\section{REFERENCES}

1 Hoo ZH, Whyte MK. Idiopathic pulmonary fibrosis. Thorax 2012;67:742-6.

2 Seibold MA, Wise AL, Speer MC, et al. A common MUC5B promoter polymorphism and pulmonary fibrosis. N Engl J Med 2011;364:1503-12.

3 Boucher RC. Idiopathic pulmonary fibrosis-a sticky business. N Engl J Med 2011;364:1560-1.

4 Stock CJ, Sato H, Fonseca C, et al. The Mucin 5B promoter polymorphism is associated with idiopathic pulmonary fibrosis but not with development of lung fibrosis in systemic sclerosis or sarcoidosis. Thorax 2013:68:436-41.

5 Zhang Y, Noth I, Garcia JG, et al. A variant in the promoter of MUC5B and idiopathic pulmonary fibrosis. N Engl J Med 2011;364:1576-7.

6 Pelito $A L$, Steele MP, Fingerlin TE, et al. The pulmonary fibrosis-associated MUC5B promoter polymorphism does not influence the development of interstitial pneumonia in systemic sclerosis. Chest 2012;142:1584-8. 